\title{
A LINEAR OPERATOR METHOD ON ARBITRARY RIEMANN SURFACES
}

\author{
BY \\ LEO SARIO
}

1. Introduction. This investigation is concerned with an operator method, which is of utility for a unified treatment of certain mapping, existence, uniqueness, and boundary value problems on Riemann surfaces.

Arbitrary Riemann surfaces $R$, closed or open, of finite or infinite genus are considered. On a compact or noncompact domain $S \subset R$ normal linear operators $L$ will be defined associating to every harmonic function $v$ in a neighborhood of the relative boundary of $S$ a uniquely determined harmonic function $L v$ on $S$. Given on $R$ a finite collection $l$ of closed curves, we select on $S=R-l$ a "singular function" $s$, that is, a real function, harmonic near $l$ and permitting a harmonic continuation across $l$. If a simple condition concerning $s$ is fulfilled, then there exists on $R$ a real function $p$, harmonic in a neighborhood of $l$ and such that

$$
p-s=L(p-s)
$$

on $S$. The function $p$ is uniquely determined and will be called the principal function for a given $s$ and $L$. If different initial functions $s$ and different operators $L$ are selected, the corresponding principal function $p(z ; s, L)$ furnishes the solution to different mapping and existence problems on $R$.

In $\$ 1$ the operator method is presented for single-valued functions. An estimation lemma is given, the above theorem is proved, and a special operator $L_{0}$ with the corresponding principal function $p_{0}$ introduced. The nature of the function $p_{0}$ is illustrated by its connection to classical slit mappings. $\$ 2$ is devoted to the existence problem of regular harmonic and analytic functions. Necessary and sufficient conditions, in terms of the normal linear operator $L_{0}$, are given. Applications to certain uniqueness questions are made. A theorem is presented for harmonic functions without existence restrictions. $\$ 3$ contains the operator method for multiple-valued functions. By way of preparation the solution of the first and second boundary value problem on abstract Riemann surfaces is given in terms of the principal function. A theorem on analytic functions without existence restrictions is proved.

In many cases the linear operator method allows, as it seems to us, a simpler approach than the Dirichlet principle or Hilbert space methods. Since all proofs are purely constructive, a numerical computation of the functions in question is possible. What is assumed a priori is the solution of the

Received by the editors June 29, 1951. 
first boundary value problem for Riemann domains with the boundary consisting of a finite number of analytic Jordan curves. As is well known this can be done simply by the Schwarz alternating method [18] which was the starting point of this investigation.

Certain results of the present paper have been announced in preliminary notes (see bibliography) $\left.{ }^{1}\right)$.

The author wishes to express his sincere gratitude to Professor Lars Ahlfors for his valuable advice in preparing this paper.

\section{§1. Operator METHOD FOR SINGLE-VALUED FUNCTIONS}

2. An estimation lemma. The Riemann surfaces to be considered may be given either in an abstract way by parameter discs or by covering or embedding properties.

The convergence proofs will be based on the following simple statement.

LEMMA 1. Let $R$ be an arbitrary Riemann surface and $K$ a closed point set on $R$. Consider on $R$ all single-valued harmonic functions $\{u\}$, normalized, by adding a suitable constant, so that

$$
\int_{K} u d \mu=0
$$

where $\mu$ is a positive mass distribution on $K$. There exists a constant $0<q<1$, independent of $u$, such that

$$
\max _{K}|u| \leqq q \cdot \sup _{R}|u|
$$

Proof. If $u$ is constant (=0), the inequality (2) is evident. The same is the case if $u$ is not bounded. Hence we can assume $\sup _{R}|u|=1$.

If (2) were not true, there would exist a sequence $\left\{u_{n}\right\}$ of functions $u$ such that

$$
\lim _{n \rightarrow \infty} \max _{K}\left|u_{n}\right|=1 \text {. }
$$

A subsequence, say again $\left\{u_{n}\right\}$, would converge towards a function $u$, harmonic and bounded, $|u| \leqq 1$, on $R$. The points where $u_{n}$ takes its maximum on $K$ accumulate at at least one point $z_{0}$ on $K$. It follows from the continuity of $u$ and the uniform convergence of $\left\{u_{n}\right\}$ on $K$ that $\left|u\left(z_{0}\right)\right|=1$. But $u$ cannot be constant, since (1) obviously is valid for it. This contradicts the maximum principle. The lemma follows.

3. Normal linear operators. By the relative boundary $l$ of a subdomain $D \subset R$ is meant the set of (inner) points of $R$ which are boundary points of $D$.

(1) The notes [8], [9], [10], where the generalization of the alternating method was given and the problem of integrals without assumption of a nullboundary was attacked, were presented to Académie des Sciences on November 28, and December 5, 1949. 
For the sake of brevity, $D$ is said in this paper to have an analytic relative boundary if $l$ consists of a finite number of disjoint analytic Jordan curves and if the complement of the closure $\bar{D}=D+l$ of $D$ has the same boundary.

A function $u$ is said to be harmonic on a curve on $R$ if it is harmonic in a neighborhood of the curve. Correspondingly, $u$ is said to be harmonic on $\bar{D}$ if it is harmonic on an open set containing $\bar{D}$. The conjugate function of a harmonic function $u$ is denoted by $\bar{u}$. For the local parameter (uniformizer) of $R$ the letter $z$ is used.

Consider linear operators with the following properties. Given a domain $D$ with an analytic relative boundary $l$ and any single-valued harmonic function $v$ on $l$, the operator $L$ defines a single-valued and unique function $L v$ on $D$; the operator is assumed to have the following properties:

$$
L v=v \text { on } l, \quad \int_{l} d \bar{L} v=0 .
$$

In addition the functional $L$ is supposed to satisfy the conditions

$$
\begin{aligned}
L 1 \equiv 1, \quad L v & \geqq 0 \text { if } v \geqq 0 \text { on } l, \\
L\left(c_{1} v_{1}+c_{2} v_{2}\right) & =c_{1} L v_{1}+c_{2} L v_{2} .
\end{aligned}
$$

An operator $L v$ satisfying (3) and (4) will be termed a normal linear operator.

4. Principal functions. Given a finite collection of disjoint Jordan curves $l$ on a Riemann surface $R$, the complement $S=R-l$ consists of a finite number of regions $S_{i}$. If in each $S_{i}$ there is given a single-valued function $s=s_{i}$, harmonic near $l$ and with a harmonic continuation across $l$, then $s$ is in general two-valued on $l$. The operation $L$ in $S$ will denote, in the following, the operation $L$ applied, in each $S_{i}$, to the branch $s=s_{i}$ defined in $S_{i}$. The two edges of $l$ are denoted by $l^{+}$and $l^{-}$, each traced in the positive direction with respect to the domains $S_{i}$ bounded by it. When integrating functions $s$ along $l^{+}$or $l^{-}$the branch of $s$ will be used which is defined in the domain $S_{i}$ bounded by this edge.

THEOREM 1. Let $R$ be an arbitrary Riemann surface, closed or open, of finite or infinite genus. Consider on $R$ a finite collection $l$ of closed curves, which is the analytic relative boundary of a compact or noncompact subregion of $R$. In $S=R-l$, let $L$ be a normal linear operator and s a single-valued real function, harmonic near $l$ and such that both branches permit a harmonic continuation across $l$. The condition

$$
\int_{l^{+}+l^{-}} d \bar{s}=0
$$

is necessary and sufficient for the existence of a single-valued real function pon $R$, harmonic on $l$ and such that in $S$ 


$$
p-s=L(p-s) \text {. }
$$

The function $p=p(z ; s, L)$ is unique $u p$ to an additive constant and will be called the principal function for given $s, L$ on $R$.

$p$ is not constant if and only if $s \neq=L s$.

Proof. By (6) and (3), it is evident that condition (5) is necessary. In order to prove the sufficiency, let $S_{0}$ be the region mentioned above of which $l$ is the relative boundary. Denote the complement $R-\bar{S}_{0}$ by $S_{1}$. Any closed curve $l_{i} \subset l$ can be mapped onto a circle $C_{i}$ in such a way that a neighborhood $T_{i}$ of $l_{i}$ is mapped conformally and biuniquely onto a neighborhood $T_{i}^{\prime}$ of $C_{i}$. Select the domains $T_{i}$ disjoint, such that $s$ is harmonic in $\bar{T}_{i}$ and such that $T_{i}^{\prime}$ is a concentric circular ring divided by $C_{i}$ into two conformally equivalent rings. Let $k$ be the boundary of $T=U T_{i}$ and let $k_{0}=k \cap S_{0}, k_{1}=k \cap S_{1}$.

Begin with the function

$$
s_{0}=s-L s
$$

in $S$ and denote by $s^{\prime}$ the harmonic function in $T$ with boundary values $s_{0}$ on $k$. The harmonic function $L s^{\prime}$ in $S$ is a linear functional $K s_{0}$ of $s_{0}$ on $k$. Write $K^{i}$ for $i$ successive operations $K$ and consider the sequence

$$
s_{n}=\sum_{i=1}^{n} K^{i} s_{0}
$$

in $S$.

Let $x$ be the harmonic function in $T$ such that $x=0$ on $k_{0}, x=2$ on $k_{1}$; then $x=1$ on $l$. Green's formula $\int(\phi d \bar{x}-x d \bar{\phi})=0$, where the integral is taken along the boundaries of $T \cap S_{0}$ and $T \cap S_{1}$, gives

$$
\int_{l} \phi d \bar{x}=\frac{1}{2} \int_{k_{0}+k_{1}} \phi d \bar{x}
$$

for any function $\phi$, single-valued on $T$, harmonic in $\bar{T}-l$, permitting a harmonic continuation from both sides over $l$, and subject to $\int_{k_{0}} d \bar{\delta}=\int_{k_{1}} d \overline{\text { }}$. Here all curves are positive in the direction which leaves $S_{0}-\left(S_{0} \cap T\right)$ to the left. From (3), (5), and the compactness of $T$ one sees that all functions in the operation $K^{i} s_{0}$ satisfy (8), so that, since $\int_{l} s_{0} d \bar{x}=0$,

$$
\int_{k_{0}+k_{1}} K^{i} s_{0} d \bar{x}=0 .
$$

In view of the construction of $K^{i}$, this implies, by Lemma 1 ,

$$
\sup _{S}\left|K^{i+1} s_{0}\right|=\max _{l}\left|K^{i+1} s_{0}\right| \leqq \max _{k}\left|K^{i} s_{0}\right| \leqq q \cdot \sup _{S}\left|K^{i} s_{0}\right| \cdot
$$

Hence the sequence (7) converges uniformly in $S$ towards a harmonic single- 
valued limit $s^{*}$ on $S$. Write

$$
p=s_{0}+s^{*} .
$$

By construction of $s_{n}$ the harmonic function in $T$ which coincides with $s_{0}+s_{n}$ on $k$ is equal to $s_{0}+s_{n+1}$ on $l$. Hence it tends, as $n \rightarrow \infty$, to the harmonic continuation of $p$, which hereby is defined on the whole surface.

Since $s^{*}$ is harmonic on $l$ (by $s^{*}=p$ ), the condition (4) yields

$$
\min _{l}\left(s^{*}-s_{n}\right) \leqq L\left(s^{*}-s_{n}\right) \leqq \max _{l}\left(s^{*}-s_{n}\right) .
$$

The difference

$$
L s^{*}-s_{n}=L s^{*}-L s_{n}=L\left(s^{*}-s_{n}\right)
$$

(where $s_{n}=L s_{n}$ by $L L=L$ ) tends to zero uniformly in $S$, hence

$$
s^{*}=L s^{*} \text {. }
$$

The condition (6) follows:

$$
p-s=s^{*}-L s=L\left(s^{*}-s+s_{0}\right)=L(p-s) .
$$

If $s \not \equiv L s$, then $p$ is not constant. In fact, the antithesis $p=$ const. $=c$ would give

$$
c-s=L(c-s)=L c-L s=c-L s,
$$

whence $s \equiv L s$. Conversely, if $s \equiv L s$, then $s_{0} \equiv 0$ and $p \equiv 0$.

The function $p=p(z ; s, L)$ is unique up to an additive constant. In order to see this, let $p_{1}$ and $p_{2}$ be two functions $p$. The difference $p_{1}-p_{2}$ is singlevalued and harmonic on $R$. On $S$, we have

$$
p_{1}-p_{2}=\left(p_{1}-s\right)-\left(p_{2}-s\right)=L\left(p_{1}-s\right)-L\left(p_{2}-s\right)=L\left(p_{1}-p_{2}\right) .
$$

Hence $p_{1}-p_{2}$ takes its maximum on $l$, and this by the maximum principle implies $p_{1}-p_{2}=$ const. This proves the theorem.

REMARK. If in the following applications of Theorem 1, the singular function $s$ is given only in some of the regions $S_{i} \subset S=R-l$, it is understood that in the remaining domains $s$ is selected $\equiv 0$.

5. The operator $L_{0}$. Different normal linear operators $L$ are defined by requiring suitable extremal properties of the function $L v$. In this paper an operator $L_{0}$ is investigated; other operators will be studied later in another connection [17].

Lemma 2. On an arbitrary Riemann surface let $S$ be a partial surface with an analytic relative boundary $l$. On $l$ is given a single-valued harmonic function $v$.

There exists on $S$ a unique harmonic function $u$ minimizing the Dirichlet integral $D_{S}[u]$ among all harmonic functions in $S$ taking boundary values $v$ on $l$, the minimum being 


$$
D_{S}[u]=\int_{l} u d \bar{u} .
$$

The function $u$ is a normal linear operator and will be noted by

$$
u=L_{0} v \text {. }
$$

The corresponding principal function will be denoted by $p_{0}(z ; s)=p\left(z ; s, L_{0}\right)$.

Proof. Let $S_{0} \subset S_{1} \subset S_{2} \subset \cdots$ be an exhaustion of $\bar{S}$ such that $S_{n}$ has an analytic relative boundary $l+k_{n}$. Let $u_{n}$ be the harmonic function in $S_{n}$ defined by $u_{n}=v$ on $l, \partial u_{n} / \partial n=0$ on $k_{n}$. $\left(u_{n}\right.$ can be constructed by well known methods, e.g. by symmetrizing $S_{n}$ and solving the first boundary value problem with values $v$ on the two copies of $l$.) Since $\min _{l} v \leqq u_{n} \leqq \max _{l} v$, a subsequence, say again $\left\{u_{n}\right\}$, converges towards a function $u$, harmonic and singlevalued in $S$. Clearly $u=v$ on $l$; hence $u$ is, by the Schwarz symmetry principle, also harmonic on $l$. The convergence of $u_{n}$ being uniform in each compact set of $\bar{S}$, it follows, by denoting $D_{n}=D_{S_{n}}$ and $D=D_{S}$,

$$
D[u]=\lim _{n \rightarrow \infty} D_{n}[u]=\lim _{n \rightarrow \infty} \lim _{m \rightarrow \infty} D_{n}\left[u_{m}\right] \leqq \lim _{m \rightarrow \infty} \int_{l} u_{m} d \bar{u}_{m}=\int_{l} u d \bar{u}<\infty .
$$

Green's formula shows immediately that $D_{n}\left[u_{n}\right]$ is the minimum $d_{n}$ of $D_{n}$ with respect to all harmonic functions in $\bar{S}_{n}$ with boundary values $v$ on $l$. Hence $d_{n} \leqq d_{n+1} \leqq d$, where $d$ is the corresponding greatest lower bound for $S, d \leqq D[u]<\infty$. By (12), the statement (10) follows.

The minimizing function $u$ is unique. In fact, if $u^{\prime}, u^{\prime \prime}$ were two functions giving the minimum $d$ for $D$, then, setting $h=u^{\prime}-u^{\prime \prime}$, clearly $D[h]<\infty$, whence $D\left[h, u^{\prime}\right]=0$. This implies that $D[h]=0$, since

$$
D\left[u^{\prime \prime}\right]=D\left[u^{\prime}\right]+D[h]+2 D\left[u^{\prime}, h\right]=D\left[u^{\prime}\right] .
$$

Hence $u^{\prime}-u^{\prime \prime}=$ const. $=0$. It follows in particular that the original sequence $\left\{u_{n}\right\}$, not merely a subsequence, converges.

It is clear, by the corresponding properties of $u_{n}$ and in view of the uniform convergence, that conditions (3) and (4) are satisfied. Hence $u$ is a normal linear operator (11).

6. Connection with slit mappings. The function $p_{0}$ is illustrated by its connection to classical slit mappings.

The principal function $p_{0}$ yields the mapping of a planar (genus =0) Riemann surface onto a plane slit domain as follows:

(1) onto a horizontal slit domain by $p_{0}+i p_{0}$ for $s=\operatorname{Re}\left(1 /\left(z-z_{0}\right)\right)$ in a disc around $z_{0}$,

(2) onto a vertical slit domain by $i p_{0}-p_{0}$ for $s=\operatorname{Im}\left(1 /\left(z-z_{0}\right)\right)$ in a disc around $z_{0}$,

(3) onto a radial slit domain by $e^{p_{0}+i \bar{p}_{0}}$ for $s=\log \left|z-z_{0}\right|$ around $z_{0}, s$ $=\log \left|z-z_{1}\right|^{-1}$ around $z_{1}$, 
(4) onto a circular slit domain by $e^{i p_{0}-\bar{p}_{0}}$ for $s=\arg \left(\left(z-z_{0}\right) /\left(z-z_{1}\right)\right)$ in $a$ disc around $z_{0}$ and $z_{1}$, cut along a curve connecting these points.

The total area of the slits vanishes.

Corresponding mappings are obtained onto circular discs or annuli with radial or circular slits. Analogous results are valid for mappings of surfaces of positive finite genus onto multiple sheeted slit-domains.

\section{§2. EXISTENCE OF REGULAR FUNCTIONS}

7. Function classes. In the present paper, only integrals are investigated, i.e. harmonic or analytic functions with a single-valued differential. The following notations will be used, for the sake of brevity, for different classes of integrals.

Greek letters $\alpha, \beta, \gamma, \delta$ will denote classes of (single- or) multiple-valued regular nonconstant harmonic functions with the following restrictive properties:
$\alpha$ all functions,
$\beta$ bounded functions,
$\gamma$ half bounded functions,
$\delta$ Dirichlet bounded functions.

The phrase "half bounded functions" is here used to mean functions bounded only upwards or only downwards, e.g. the positive functions. (The letter $\gamma$ can be interpreted in certain analogy to Green's functions.) For a multiplevalued function, the properties $\beta$ and $\gamma$ have to be understood in the sense that the existence domain considered can be cut along such curves that the function is single-valued in the cut domain and has there the property $\beta$ or $\gamma$, respectively. "Dirichlet bounded" signifies that the functions have a finite Dirichlet integral.

By a dividing cycle is meant a finite set of simple curves dividing the given Riemann surface in two separate parts. The subclasses

$$
\alpha_{0} \subset \alpha, \quad \beta_{0} \subset \beta, \quad \gamma_{0} \subset \gamma, \quad \delta_{0} \subset \delta
$$

denote classes of functions $\alpha, \beta, \gamma, \delta$ with vanishing periods along each dividing cycle. This property could be expressed by saying that the function has no periods along any cycle around a boundary part.

The classes of single-valued regular nonconstant harmonic functions will be denoted by the corresponding Latin letters $a, b, g, d$,

$$
a \subset \alpha_{0}, \quad b \subset \beta_{0}, \quad g \subset \gamma_{0}, \quad d \subset \delta_{0} .
$$

For planar surfaces, clearly,

$$
a=\alpha_{0}, \quad b=\beta_{0}, \quad g=\gamma_{0}, \quad d=\delta_{0} .
$$


The classes of regular analytic functions will be denoted by pairs of letters, the first expressing the class of the real part, the second the class of the imaginary part. E.g.:

$\beta_{0} d$ regular analytic functions with a bounded real part without periods along dividing cycles and a single-valued imaginary part having a finite Dirichlet integral.

Given an open Riemann surface $R$, the complement $R-\bar{D}$ of any compact domain $\bar{D}$ with an analytic boundary $l$ will be called a boundary neighborhood $N$ of $R$. A property of $R$ will be termed a boundary property if it can be expressed as a property of an arbitrarily small boundary neighborhood $N$, i.e. of the complement of a domain $\bar{D}$ tending to $R$.

In the following sections the existence of functions on $R$ is investigated in terms of its dependence on the boundary. Relations between different classes will be considered in subsequent papers [16].

We note first:

Given on an arbitrary Riemann surface a domain $D$ with analytic relative boundary $l$, and on $l$ any single-valued harmonic function $v$, then on $D$

$$
L_{0} v \in b, g, d, \quad \bar{L}_{0} v \in \alpha_{0} .
$$

On domains of finite genus

$$
\bar{L}_{0} v \in \beta_{0}, \gamma_{0}, \delta_{0}
$$

On planar domains

$$
L_{0}+i \bar{L}_{0} \in f f^{\prime}
$$

where $f$ and $f^{\prime}$ may be any two of the symbols $b, g, d$.

8. Existence of harmonic functions. Given a class $f$ of functions, we denote by $0_{f}$ the class of Riemann surfaces on which there are no functions belonging to the class $f$. Now let $R$ be an arbitrary Riemann surface. Consider first the existence of single-valued harmonic functions on $R$.

TheOREM 2. A necessary and sufficient condition for

$$
R \in 0_{f}, \quad f=b, g, d,
$$

is that in some boundary neighborhood $N$ each function $\phi \in f$, satisfying $\int_{l} d \bar{\phi}=0$, is invariant under the operation $L_{0}$ :

$$
\phi \equiv L_{0} \phi
$$

This condition is independent of $N$, i.e. (19) is a boundary property.

The condition (20) is, for $f=b, d$, equivalent to the following: for any two functions $\phi_{1}, \phi_{2}, \in f$ in $N$, resp. $\phi_{1}-L_{0} \phi_{1}$ and $\phi_{2}-L_{0} \phi_{2}$ are linearly dependent.

Proof. If there exists on $N$ a function $\phi \in f, \int_{l} d \phi=0, \phi \neq \equiv L_{0} \phi$, then, by Theorem 1 and (17), there is on $R$ a function $\phi \in f$. Conversely, for a func- 
tion $\phi \in f$ on $R$ clearly $\int_{l} d \phi=0$ and, by the maximum principle,

$$
\sup _{R} \phi=\sup _{N} \phi>\max _{l} \phi,
$$

whence $\phi \not \equiv L_{0} \phi$.

For proving the second statement assume there exist on $N$ two functions $\phi_{1}, \phi_{2} \in f$ such that $\phi_{1}-L_{0} \phi_{1}$ and $\phi_{2}-L_{0} \phi_{2}$ are linearly independent. Clearly

$$
\phi_{1} \not \equiv L_{0} \phi_{1}, \quad \phi_{2} \not \equiv L_{0} \phi_{2} .
$$

If $\int_{l} d \bar{\phi}_{1}=0$ or $\int_{l} d \bar{\phi}_{2}=0$, then the existence of $\phi \in f$ on $R$ is guaranteed by Theorem 1. If both integrals are $\neq 0$ then, for a suitable real constant $\lambda$, the function $\psi=\left(\phi_{1}-L_{0} \phi_{1}\right)+\lambda\left(\phi_{2}-L_{0} \phi_{2}\right)$ satisfies $\int_{l} d \psi=0, \psi \neq \equiv L_{0} \psi$ in $N$, and there exists a $\phi \in f$ on $R$.

Conversely, assume there is a $\phi \in f$ on $R$. In $N$ the function $\phi^{\prime}=\phi-L_{0} \phi \in f$ is not constant and $\int_{l} d \bar{\phi}^{\prime}=0$. Hence $\phi^{\prime}$ takes in $N$ both positive and negative values. Take an exhaustion $N_{n}$ of $N$, the boundary $l+k_{n}$ of $N_{n}$ being analytic. If $\phi \in b, \phi^{\prime} \leqq M<\infty$, let $\psi_{n}$ be the harmonic function in $N_{n}$ with $\psi_{n}=0$ on $l, \psi_{n}=M$ on $k_{n}$. Since $\left\{\psi_{n}\right\}$ is a normal family, some limit function $\phi^{\prime \prime}$ in $N$ satisfies $\phi^{\prime \prime} \in b, \phi^{\prime \prime} \geqq \phi^{\prime}, \phi^{\prime \prime} \geqq 0$, hence $\int_{l} d \bar{\phi}^{\prime \prime} \neq 0$. The functions $\phi^{\prime}=\phi^{\prime}-L_{0} \phi^{\prime}$ and $\phi^{\prime \prime}=\phi^{\prime \prime}-L_{0} \phi^{\prime \prime}$ are linearly independent. An analogous reasoning proves the case $\phi \in d$ (cf. no. 11 in [6]).

For multiple-valued harmonic functions the existence theorem is:

THEOREM 3. On every Riemann surface of positive (finite or infinite) genus there are functions $\beta_{0}, \gamma_{0}, \delta_{0}$. On a planar surface $R$, these functions exist if and only if $R$ is hyperbolic.

Proof. If there is a nondividing cycle $k$ on $R$, divide it into two parts $k_{1}$ and $k_{2}$. Let $D$ be a compact domain containing $k_{1}$. Take in $\bar{D}$ for $s$ a harmonic function with the singularities arg $\left(z-z_{1}\right)$ and $-\arg \left(z-z_{2}\right)$ at the end points $z_{1}, z_{2}$ of $k_{1}$ respectively. Let $p_{1}=p_{0}(z ; s)$ be the corresponding principal function on $R$. Then take around $k_{2}$ for $s$ a harmonic function with the singularities arg $\left(z-z_{2}\right)$ and $-\arg \left(z-z_{1}\right)$ at $z_{2}$ and $z_{1}$ respectively. Denote by $p_{2}$ the resulting principal function $p_{0}(z ; s)$. Then $p=p_{1}+p_{2} \in \beta_{0}, \gamma_{0}, \delta_{0}$ on $R$.

Assume now $R$ is planar. Then $\beta_{0}=b, \gamma_{0}=g, \delta_{0}=d$ and the nonexistence is implied by condition (20). It is easy to see that this is, for vanishing genus, equivalent with $R$ being of parabolic type.

9. Existence of analytic functions. Consider first multiple-valued analytic functions. The proofs of Theorems 2 and 3 yield immediately:

THEOREM 4. There are functions $f f^{\prime}$ for

$$
f=b, g, d, \quad f^{\prime}=\alpha, \alpha_{0}
$$

on an arbitrarily given Riemann surface if and only if in some boundary neighborhood $N$ there is a function $\phi+i \bar{i} \in f f^{\prime}$, subject to $\int_{l} d \phi=0$, such that 


$$
\phi \not \equiv L_{0} \phi \text {. }
$$

On every Riemann surface of positive (finite or infinite) genus there are functions $f^{\prime}$ for

$$
f=\beta_{0}, \gamma_{0}, \delta_{0}, \quad f^{\prime}=\alpha, \alpha_{0} .
$$

On a planar surface these functions $f f^{\prime}$, for $f^{\prime}=\alpha$, exist if and only if the surface is of hyperbolic type.

For $f^{\prime}=\alpha_{0}$, the latter functions are single-valued and subject to Theorem 5 below.

Consider now the existence of single-valued analytic functions. The boundary $r$ of a Riemann surface $R$ is said to be removable $f^{\prime}$ if there are no analytic functions belonging to the class $f f^{\prime}$ on $R$ [6].

THEOREM 5. The boundary $r$ of a Riemann surface $R$ of finite genus is removable ff',

$$
R \in 0_{f f^{\prime}}, \quad f=b, d, \quad f^{\prime}=a, b, d,
$$

if and only if in some boundary neighborhood $N$ for each function $\phi+i \bar{\phi} \in f f^{\prime}$

$$
\phi \equiv L_{0} \phi .
$$

Proof. Suppose there is a function $\phi+i \phi \in f f^{\prime}$ on $N, \phi \neq \equiv L_{0} \phi$. Take a planar boundary neighborhood $N_{0} \subset N$, which can be mapped conformally and biuniquely onto parts $N^{\prime}$ of circular discs $K$ in such a way that the relative boundary $l$ of $N_{0}$ goes onto the boundary circles $l^{\prime}$ of $K$. The map of the boundary $r$ of $R$ is some point set $r^{\prime} \subset K$. If $r^{\prime}$ contains a proper continuum, then the existence of a function $f f^{\prime}$ on $R$ is clear. Suppose $r^{\prime}$ is totally disconnected. The function $\phi_{0}=\phi-L_{0} \phi$ in $N$ is, by (17)', subject to the condition $\phi_{0}+i \bar{\phi}_{0} \in f f_{0}^{\prime}$, where $f_{0}^{\prime}=\alpha_{0}, \beta_{0}, \delta_{0}$ for $f^{\prime}=a, b, d$ respectively. Hence by (18) each branch of $\phi_{0}+i \bar{\phi}_{0} \in f f^{\prime}$ on $N_{0}$. This implies that $\phi_{0}$ has an infinite set $\rho$ of singular points on $r^{\prime}$, since otherwise $\phi_{0} \equiv 0$.

Let $l_{i}, i=1, \cdots, 2 q+1$ ( $q$ =genus of $R$ ), be analytic curves in $N_{0}$ bounding disjoint simply-connected domains $S_{i} \subset K$, each containing some subset $\rho_{i} \subset \rho$. To the function $s_{i}=\phi_{0} \not \equiv L_{0} \phi_{0}$ on $S_{i}$ corresponds a nonconstant principal function $p_{i}=p_{0}\left(z ; s_{i}\right)$ on the closed surface $R^{*}=R-N_{0}+K$ such that $p_{i}+i \bar{p}_{i} \in f f_{0}^{\prime}$. For any real constants $c_{i}$,

$$
p=\sum_{i=1}^{2 q+1} c_{i} p_{i} \neq \text { const. }
$$

on $R^{*}$ since $p$ has singular points. Select the constants $c_{i}$ so as to satisfy

$$
\sum c_{i} \int_{\alpha_{k}} d \bar{p}_{i}=0
$$


along each of the $2 q$ nondividing canonical cycles $\alpha_{k}$ of $R$. Then $p+i \not p \in f f^{\prime}$ on $R$.

Conversely, assume that there is a function $\phi+i \phi \in f f^{\prime}$ on $R$. Then in $N_{0}, \phi_{0}=\phi-L_{0} \phi \neq 0$. This completes the proof.

REMARKS. (1) In case of an infinite genus, the removability with respect to single-valued analytic functions is a relative property. It was shown by Ahlfors [2] that there are boundary neighborhoods $N$ which can be completed, by adding along the relative boundary $l$ two different compact parts $D_{1}$ or $D_{2}$, to two different Riemann surfaces $R_{1}$ or $R_{2}$ such that $R_{1} \in 0_{f f^{\prime}}, R_{2} \notin 0_{f f^{\prime}}$. A statement corresponding to Theorem 5 can therefore not hold for surfaces of infinite genus.

(2) For surfaces $R$ of finite genus, the notion of a removable boundary $f f^{\prime}$ coincides with the classical notion of a removable singularity. In fact, the boundary of $R$ being realized as a point set $r$ in a circle $K, r$ is a removable singularity for each function $f f^{\prime}$ in $K-r$ if and only if there are no functions $f f^{\prime}$ on $R$. This is implied in the above reasoning for any class $f f^{\prime}$ with $f=b, d$; $f^{\prime}=\alpha, a, b, d$.

The removability of the boundary is closely related to certain uniqueness questions:

$A$ Riemann surface $R$ of finite genus $\in 0_{f^{\prime}}\left(f, f^{\prime}=b\right.$ or $\left.d\right)$ possesses the following properties:

$1^{\circ}$. All closed extensions of $R$ are conformally equivalent.

$2^{\circ}$. The conformal mapping of $R$ onto a part of a closed surface $R^{*}$ is unique up to a transformation of $R^{*}$ onto itself. In particular:

$3^{\circ}$. A plane mapping of a planar surface $R$ is unique up to a linear transformation. Hence:

$4^{\circ}$. All uniformizations of a Riemann surface by a (fixed) planar covering surface $R$ depend linearly on each other.

These statements are an immediate consequence of Theorem 5 and remark (2).

10. Functions without existence restrictions. As a supplement to Theorem 1, we prove first:

Lemma 3. On an open Riemann surface $R$, consider a noncompact domain $D$ with analytic relative boundary $l$. Given an arbitrary finite real number $c$ there always exists a single-valued harmonic function $u$ on $D$ such that

$$
\int_{l} d \bar{u}=c .
$$

Proof. For $c=0$ the statement is proved by $u \equiv 0$. For $c \neq 0$ take an exhaustion $\left\{D_{n}\right\}$ of $D, D_{n}$ having an analytic relative boundary $l+k_{n}$. Let $u_{n}$ be the harmonic function in $D_{n}$ such that $u_{n}=0$ on $l, u_{n}=$ const. on $k_{n}, u_{n}=1$ at a fixed point $z_{0} \in D_{0}$. For the functions $u_{n+p}(p=1,2, \cdots)$, positive on $D_{n+1}$, 
the Harnack principle yields, on the compact point set $z_{0} \cup k_{n}$,

$$
u_{n+p}(z)<M \cdot u_{n+p}\left(z_{0}\right)=M,
$$

where $M$ is a constant $<\infty$ independent of $p$. Hence $u_{n+p}$ is a normal family and there exists on $D$ a single-valued harmonic limit function $u$. Clearly $u=0$ on $l, u=1$ at $z_{0}, u>0$ on $D$, hence $\int_{\imath} d \bar{u}>0$. By multiplying $u$ by a suitable constant the lemma follows.

THEOREM 6. Let $R$ be an arbitrary open Riemann surface and $D$ a noncompact domain $(D \subset R)$ with analytic relative boundary $l$. In $S=R-\bar{D}$ let $s$ be a single-valued real function, harmonic on $l$. Given on $S$ a normal linear operator $L$ there is on $R$ a single-valued function $p$, harmonic on $\bar{D}$ and such that, on $S$,

$$
p-s=L(p-s) .
$$

Proof. By the previous lemma there is a single-valued harmonic function $u$ on $D$ such that

$$
\int_{l} d \bar{u}=\int_{l} d \bar{s}
$$

The assumptions of Theorem 1 are thereby satisfied in $R-l$ and the truth of the statement follows.

§3. Operator method for multiple-valued functions

11. The first and second boundary value problem. Consider on an arbitrary Riemann surface a compact domain $D$ with an analytic boundary $l$. Let $v$ be a harmonic single-valued function on $l$. Denote by $D^{*}$ the symmetrized domain $D$, by $z_{0}, z_{0}^{*}$ two symmetric points, by $K, K^{*}$ circular discs around them.

The principal function $p_{0}(z ; s)$ on $D^{*}$ for $s=\log \left|z-z_{0}\right|^{-1}$ in $K$, $s$ $=\log \left|z-z_{0}^{*}\right|$ in $K^{*}$, is the Green's function of $D$ with the logarithmic pole at $z_{0}$ and gives the solution $u$ to the Dirichlet problem with boundary values $v$ :

$$
u\left(z_{0}\right)=-\frac{1}{2 \pi} \int_{l} v d \Phi_{0}(z ; s) .
$$

The principal function $p_{0}(z ; s)$ on $D$ for $s=\log \left|z-z_{0}\right|^{-1}$ around $z_{0}$, $s=\log \left|z-z_{1}\right|$ around $z_{1}$ is a generalized Neumann's function with positive and negative poles at $z_{0}$ and $z_{1}$ respectively and with a vanishing normal derivative on $l$. It gives for the Neumann problem the solution $u$ which vanishes at $z_{1}$,

$$
u\left(z_{0}\right)=-\frac{1}{2 \pi} \int_{l} p_{0}(z ; s) \frac{\partial v}{\partial n} d s .
$$


Both statements are immediately derived from Green's formula applied to $D$ less small circular discs centered at the points $z_{0}$ and $z_{1}$.

The relation (25) will now furnish the solution of the first boundary value problem for a multiple-valued $v$ :

Lemma 4. Let $v$ be a harmonic (in general multiple-valued) function on $l$. The condition

$$
\int_{l} d v=0
$$

is necessary and sufficient for the existence of a harmonic function on $D$ coinciding with $v$ on $l$.

Proof. Let $u$ be a harmonic function with normal derivative $\partial u / \partial n$ $=-\partial v / \partial s$ on $l$, where $\partial n$ is taken in the inner direction of $D, \partial s$ in the positive direction along $l$ with respect to $D$. Then $\bar{u}$ is a harmonic function in $D$ with $\partial \bar{u} / \partial s=\partial v / \partial s$ on $l$, hence $\bar{u}=v+c_{i}$ on the closed curves $l_{i} \subset l\left(c_{i}=\right.$ const.). Let $h$ be the harmonic function in $D$ such that $h=c_{i}$ on $l_{i}$. Then $\bar{u}-h$ is the desired function.

Clearly the condition (26) is necessary.

12. The generalized operator method. Theorem 1 has the following counterpart for (in general multiple-valued) analytic functions:

THEOREM 7. Consider an arbitrary Riemann surface $R$ and a compact domain $D$ on $R$ with an analytic boundary $l$. Let $L$ be a normal linear operator in $S=R-\bar{D}$ and let $\sigma=s+i t$ be a complex-valued function on $\bar{S}$, analytic, $\sigma=s+i \bar{s}$, on $l$. The condition

$$
\int_{l} d \sigma=0
$$

is necessary and sufficient for the existence of a complex-valued function $w=p$ $+i q$ on $R$, analytic on $\bar{D}, w=p+i \bar{p}$, and such that in $S$

$$
p-s=L(p-s) \text {. }
$$

If $s \not \equiv L s$ then $w \not \equiv$ const.

Proof. Let $T(\supset \bar{D})$ be a compact domain with an analytic boundary $k$ and such that $\sigma$ is analytic in $\bar{G}=\bar{T} \cap \bar{S}$. By Lemma 4, there is a harmonic function $s^{\prime}$ in $T$ coinciding with $s$ on $k$. The difference $s^{\prime}-s$ is single-valued in $\bar{G}$. Write, in $S$,

$$
s_{0}=L\left(s^{\prime}-s\right)
$$

Let $s^{\prime \prime}$ be the harmonic function in $T$ with the boundary values $s_{0}$ on $k$. Then, in $S, L s^{\prime \prime}$ is a linear operator $K s_{0}$ of $s_{0}$ on $k$. Consider the sequence 


$$
s_{n}=\sum_{i=0}^{n} K^{i} s_{0}
$$

$\left(K^{0} s_{0}=s_{0}\right)$ in $S$.

For any single-valued harmonic function $\psi$ in $\bar{G}$, subject to the condition $\int_{l} d \Psi=0$, clearly

$$
\int_{l} \psi d \bar{x}=\int_{k} \psi d \bar{x}
$$

where $x$ is the harmonic measure of $k$ in $\bar{G}$. It follows, by $\int_{k}\left(s^{\prime}-s\right) d \bar{x}=0$ and the compactness of $T$, that

$$
\int_{k} K^{i} s_{0} d \bar{x}=0
$$

Hence Lemma 1 is applicable, and the same reasoning as in no. 4 yields the uniform convergence of the sequence (30) towards a harmonic limit $s^{*}$ in $\bar{S}$. The harmonic function in $T$ with boundary values $s+s_{n}$ on $k$ converges uniformly towards the harmonic continuation of $p=s+s^{*}$. Hence

$$
w=p+i\left(t+s^{*}\right)
$$

is the desired function on $R$.

13. Unrestricted existence of analytic functions. We close by giving a supplement to Theorem 7.

LEMma 5. Consider on an open Riemann surface a noncompact domain $S$ with an analytic relative boundary $l$. Given an arbitrary finite complex number $c_{1}+i c_{2}$, there always exist analytic functions $\tau$ (with single-valued differentials) on $S$ such that

$$
\int_{l} d \tau=c_{1}+i c_{2}
$$

Proof. Let $u_{1}$ and $u_{2}$ be two single-valued harmonic functions in $\bar{S}$ such that $\int_{l} d \bar{u}_{1}=c_{1}$ and $\int_{l} d \bar{u}_{2}=c_{2}$. Then $\tau=\left(\bar{u}_{1}+u_{2}\right)+i\left(\bar{u}_{2}-u_{1}\right)$ is the desired function.

TheOREM 8. On an arbitrary open Riemann surface $R$ let $D$ be a compact domain with an analytic relative boundary. Let $S_{0}$ and $S_{1}$ be disjoint collections of domains which constitute $R-\bar{D}\left(R=\bar{D}+S_{0}+S_{1}\right)$. In $S_{0}$, let $\sigma=s+i t$ be a complex-valued function, analytic, $\sigma=s+i \bar{s}$, on the relative boundary $l_{0}$ of $S_{0}$. Given on $S_{0}$ a normal linear operator $L$, there exists a complex-valued function $w=p+i q$ on $R$, analytic on $S_{1}+\bar{D}, w=p+i \bar{p}$, and such that on $S_{0}$

$$
p-s=L(p-s) \text {. }
$$


Proof. By Lemma 5, there is an analytic function $\tau$ on $S_{1}$ such that

$$
\int_{l_{0}} d \sigma+\int_{l_{1}} d \tau=0
$$

where $l_{1}$ is the relative boundary of $S_{1}$. The statement then follows by Theorem 7 .

\section{BIBLIOGRAPHY}

1. L. Ahlfors, Open Riemann surfaces and extremal problems on compact subregions, Comment. Math. Helv. vol. 24 (1950) pp. 100-134.

2. - Remarks on the classification of open Riemann surfaces, Annales Academiae Scientiarum Fennicae Ser. A, I. Mathematica-Physica no. 87 (1951) pp. 1-8.

3. L. Ahlfors and A. Beurling, Conformal invariants and function-theoretic null-sets, Acta Math. vol. 83 (1950) pp. 101-129.

4. H. Royden, Some remarks on open Riemann surfaces, Annales Academiae Scientiarum Fennicae Ser. A, I. Mathematica-Physica no. 85 (1951) pp. 1-8.

5. L. Sario, Ueber Riemannsche Flächen mit hebbarem Rand, Annales Academiae Scientiarum Fennicae Ser. A, I. Mathematica-Physica no. 50 (1948) pp. 1-79.

6. - Sur la classification des surfaces de Riemann, XI Congr. Math. Scand., Trondheim, 1949, pp. 229-238.

7. - Sur le problème du type des surfaces de Riemann, C. R. Acad. Sci. Paris vol. 229 (1949) pp. 1109-1111.

8. - Existence des fonctions d'allure donnée sur une surface de Riemann arbitraire, C. R. Acad. Sci. Paris vol. 229 (1949) pp. 1293-1295.

9. - Quelques propriélés à la frontière se rattachant à la classification des surfaces de Riemann, C. R. Acad. Sci. Paris vol. 230 (1950) pp. 42-44.

10. — Existence des intégrales abéliennes sur les surfaces de Riemann arbitraires, $\mathrm{C} . \mathrm{R}$. Acad. Sci. Paris vol. 230 (1950) pp. 168-170.

11. - Questions d'existence au voisinage de la frontière d'une surface de Riemann, C. R. Acad. Sci. Paris vol. 230 (1950) pp. 269-271.

12. - On open Riemann surfaces, International Congress of Mathematicians, Cambridge, 1950 . 304.

13. - Linear operators on Riemann surfaces, Bull. Amer. Math. Soc. Abstract 57-4-

14. - Principal functions on Riemann surfaces, Bull. Amer. Math. Soc. Abstract $57-6-492$

15. - Existence of functions as a boundary property, Bull. Amer. Math. Soc. Abstract 58-1-23.

16. - An extremal method on arbitrary Riemann surfaces, Trans. Amer. Math. Soc. (to appear).

17. - Construction of functions with prescribed properties on Riemann surfaces, Conference on Riemann Surfaces in Commemoration of the Hundredth Anniversary of Riemann's Inaugural Dissertation, Princeton, 1951, Annals of Mathematics Studies (to appear).

18. H. A. Schwarz, Ges. Math. Abh., Berlin 1890, vol. II, pp. 133-171, 303-306, 356-358, $360-362$.

The Institute for Advanced Study,

Princeton, N. J. 